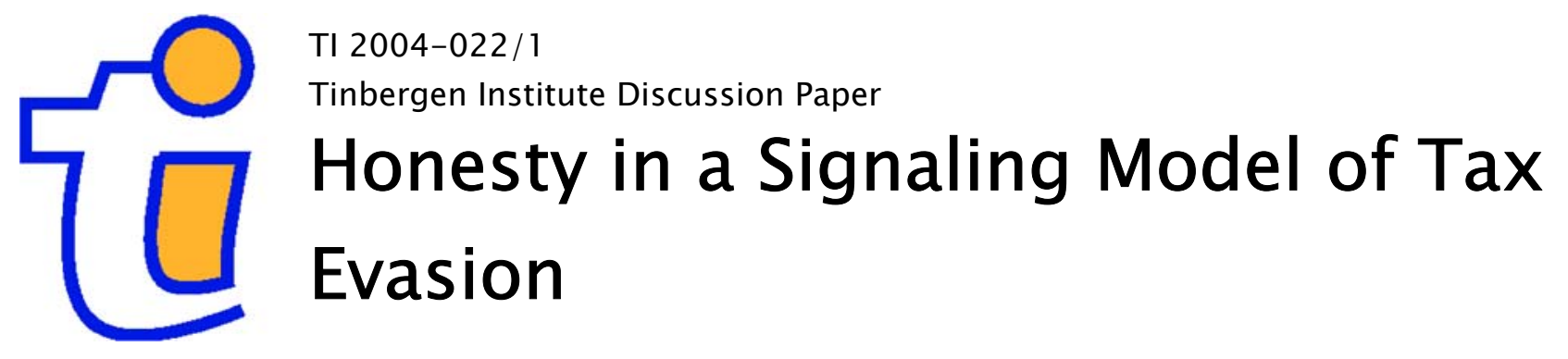

Vitaly Pruzhansky

Department of Econometrics, Faculty of Economics and Business Administration, Vrije Universiteit Amsterdam, and Tinbergen Institute. 


\section{Tinbergen Institute}

The Tinbergen Institute is the institute for economic research of the Erasmus Universiteit Rotterdam, Universiteit van Amsterdam, and Vrije Universiteit Amsterdam.

Tinbergen Institute Amsterdam

Roetersstraat 31

1018 WB Amsterdam

The Netherlands

Tel.: $\quad+31(0) 205513500$

Fax: $\quad+31(0) 205513555$

Tinbergen Institute Rotterdam

Burg. Oudlaan 50

3062 PA Rotterdam

The Netherlands

Tel.: $\quad+31(0) 104088900$

Fax: $\quad+31(0) 104089031$

Please send questions and/or remarks of nonscientific nature to driessen@tinbergen.nl.

Most TI discussion papers can be downloaded at http://www.tinbergen.nl. 


\title{
HONESTY IN A SIGNALING MODEL OF TAX EVASION
}

\author{
VITALY PRUZHANSKY
}

\begin{abstract}
We study the phenomenon of tax evasion using a simple signaling model, in which the signal is taxpayer's reported income. The novelty of our approach lies in the way we define honesty. Specifically, we advocate the view that there are no absolutely honest taxpayers: all taxpayers may under certain conditions become evaders. We investigate the implications of this type of honesty on the equilibria of the model, and compare it to the basic case, in which honesty is absent. Our analysis completely characterizes all perfect Bayesian Nash equilibria of the game, assesses their stability and welfare properties.
\end{abstract}

\section{INTRODUCTION}

In a historical perspective the theoretical analysis of tax evasion can be best described by dividing all existing models into two groups: first and second generation. The classical literature of the first generation models studied a taxpayer who solved a gambler's problem on how much to evade, given an exogenous tax rate, penalty for tax evasion and detection rate. The earliest formal analyses were given by Allingham and Sandmo [1], Srinivasan [17], Yitzhaki [22], who analyzed how the changes of the above parameters affected evaded income. Their approaches differed only in the structure of punishments and assumptions about taxpayer's attitude towards risk. One common conclusion of all these models was that the size of penalty and detection rate are substitutes, however increasing penalty is costless, whereas increasing the probability of detection

Date: February 13, 2004.

Key words and phrases. Auditing, equilibrium refinements, tax morale, underground economies.

A significant part of this paper was completed while a student at the Central European University, Hungary in 2000. I am thankful to prof. L. Ambrus-Lakatos and to my former colleague P. Grishine. Comments of J. Kamphorst and G. van der Laan are gratefully acknowledged. The usual disclaimer applies. 
involves additional resources. Based on this, it led to the following policy recommendation: in order to maximize tax revenues minus costs of audits one should set an infinite fine and zero detection rate.

It is essential to note that all first generation models were essentially decision, not game-theoretic, and thus disregarded the effects of the actions of one party on the other. Specifically, the above policy recommendation did not constitute a Nash equilibrium, as is easy to check. Despite this, we believe, quite serious drawback ${ }^{1}$, first generation models of tax evasion, for some poorly understood reasons, are still being tested empirically and cited without even mentioning this fact, eg. Frey [7], Torgler [19] and [20]. The rationale for doing so is very obscure to us!

Second generation models of tax evasion (eg. Erard and Feinstein [5], Graetz et al. [6], Reinganum and Wilde [13] and [14]) have adopted a much more realistic, interactive, view of the tax collecting process. Specifically, an independent tax collecting agency was included into the analysis, whose objective function was to maximize tax proceedings net auditing costs, given exogenously specified tax and penalty rates. Models of the second generation explicitly used game-theoretic tools and looked for Nash equilibria of the corresponding tax evasion game. The main conclusion of this line of research was that the optimal auditing policy (probability of detection) negatively depended on the amount of reported income and that tax evasion declined with the rise of the taxpayer's income.

Neither first, nor second generation models performed satisfactory during empirical testing. In particular, they predicted much more tax evasion than was observed in reality. To cite just one example, Frey [7] (while testing a first generation model on the data from Swiss cantons) reports '...a coefficient of relative risk aversion of 30.8 would be necessary in order to achieve the compliance rate of 76.5 percent...'. Empirical studies of risk aversion, however, suggest that the latter coefficient is between 1 and 2. With the purpose of achieving a better fit to the data, existing models were modified. For instance, it was suggested that considerations other than simple utility maximization, like honesty, fairness, responsibilities of citizenship and the like also influence taxpayers' decision to pay honestly. The combination of all these factors has been dubbed tax morale and claimed to be one of the most decisive factors stimulating taxpayers' compliance.

Positive effects of tax morale can certainly be included in the analysis in many different ways. For example, following Frey [7], one can argue that

\footnotetext{
${ }^{1}$ See Section $5 \mathrm{j}$ for a discussion of how significant this issue is.
} 
tax officials are aware of the positive effects of tax morale on taxpayers' compliance, and, hence, treat taxpayers respectfully, so that morale is not destroyed; as a consequence, tax revenues increase. In this paper we propose a simpler and more intuitive approach. We associate tax morale with inner honesty. The latter is defined as a force that may keep taxpayers from misreporting their income in situations, when there are positive gains from evasion in expected utility terms ${ }^{2}$. We then compare a basic model, in which taxpayers are not honest in the above sense, with the one, where honesty is in place. Expected utilities of both the agency and taxpayers across all possible Nash equilibria are computed and compared. One of our conclusions states that audits are not socially optimal, in the sense that all parties could be made strictly better off by boosting taxpayers' honesty. We do not discuss how exactly this can be done, though. Respectful treatment of taxpayers offered in Frey [7] can well serve as an example of such a tool.

To derive our results we use a simple signaling model, in which the signal is the taxpayer's reported income. As it is typical for this type of models, multiple equilibria arise. To test their reasonableness we employ the intuitive criterion developed in Cho and Kreps [4], as well as evolutionary and stochastic stability. It will be shown that all these refinements favor the same equilibrium, in which all taxpayers report their income honestly. Another interesting conclusion is that the only equilibrium, in which the agency conducts audits, is dynamically unstable, since it involves mixed strategies. Despite its simplicity, we believe that our modelling approach can be relevant to a wide range of situations, in which cheating and punishments are not certain. (As an example, consider payments for using public transport).

The current paper is similar in spirit to the models of the second generation. However, contrary to them, we take up a local, not global perspective. We do not aggregate all taxpayers in one population, some fraction of which is always assumed to report honestly, whereas the members of the other one are expected utility maximizers. Nor do we deal with such things as budget constraints of the tax agency, which has to extract maximal amount of taxes from the population of taxpayers, given a fixed budget. Our approach is restricted to one tax collector and a representative taxpayer. Furthermore, the word 'representative', does not refer to the whole population of taxpayers. It is interpreted in a much narrower sense, say, a representative of a particular income category or

\footnotetext{
${ }^{2}$ Cf. the work of Erard and Feinstein [5], who assumed the existence of inherently honest taxpayers, who always report their income truthfully.
} 
business firm of a certain size or type. Bearing these points in mind is crucial for correct interpretation of our results, as is discussed in Section $5 \mathrm{k}$.

Another important distinction between the framework we propose and other models of the second generation is that our analysis is carried out from a positive, not normative standpoint. Although it was forcefully argued by Friedman [8] that the usefulness of a positive model can only be judged by comparing its theoretical predictions with empirical data, we do not make any empirical comparisons in this paper. In fact, we take empirical evidence on the high level of compliance as given. Nor do we intend to 'explain' tax evasion with the help of this model by claiming that it fits the data. What our model tries to achieve is to capture some 'hidden', not immediately obvious, relationships behind the process of tax collection and assess the welfare properties of stable, equilibrium situations. In this regard, we believe, we are still in line with Friedman's methodology ${ }^{3}$.

The paper is structured as follows. Section 2 presents a basic model, and Section 3 considers an extended version, in which honesty of taxpayers is explicitly taken into account. Section 4 applies various equilibrium refinements to the results of Section 3. Section 5 discusses interpretation and possible extensions of the model.

Throughout it is assumed that the reader is familiar with the standard game-theoretic terminology, such as perfect Bayesian equilibria and refinements thereof, evolutionary and stochastic stability ${ }^{4}$. Note that all perfect Bayesian equilibria in this paper can be shown to be equivalent to the stronger notion of sequential equilibrium that is the principal solution concept for the class of extensive form games of imperfect information, to which our model belongs. We have opted for perfect Bayesian equilibria because they are easier to work with ${ }^{5}$, and because in the present setup it involves no loss of generality.

\section{The Basic Model}

There are two players in the game: the taxpayer (player 1) and the tax agency (player 2). At the beginning of the game Nature selects the

\footnotetext{
${ }^{3}$ Such an approach is not uncommon. For instance, the Solow-Swan model of economic growth (see eg. Barro and Sala-i-Martin [3]) has the same descriptive character.

${ }^{4}$ For an excellent introduction to these topics see Osborne and Rubinstein [11] and references therein. Young [23] is a classical source on stochastic stability.

${ }^{5}$ More specifically, a technical procedure of checking the consistency of players' beliefs can be avoided.
} 
type of the taxpayer, $\tau \in\{Y, y\}$. Here $Y$ and $y$ are two distinct levels of income, such that $Y>y$; sometimes we will call them 'high' and 'low', respectively. The taxpayer, having observed his type, chooses whether to report it to the agency honestly or not. Finally, the agency, having observed the report, but not the type of the taxpayer, decides whether to audit the latter or not. These actions are denoted, respectively, by $a$ and $d$. It is common knowledge between the players that Nature selects $\tau=Y$ with probability $\pi$, and that $Y$ and $y$ are the only types of income that can be obtained and declared (see Sections $5 \mathrm{a}$ and $5 \mathrm{~g}$ for explanations and extensions).

Throughout the term cheating applies to any situation, in which the taxpayer of type $\tau^{\prime}$ reports $\tau^{\prime \prime} \neq \tau^{\prime}$. Correspondingly, there are two cases: when the taxpayer understates his income (i.e. type $Y$ reports $y$ ), which represents the instance of evasion, and when the taxpayer over-reports (i.e. type $y$ declares $Y)^{6}$. Although it is somewhat counter-intuitive to call the latter case 'cheating', for the sake of consistency we do so. All reported income is taxed at the rate $t>0$. During the process of auditing, taxpayer's true income is figured out with probability one. If the taxpayer who understated his income has been caught, he is required to pay the missing amount of the tax, $(Y-y) t$, plus a fine, whose size is proportional to the amount of income evaded and equals $(Y-y) \theta$ for some $\theta>0$. If the taxpayer over-reports and is subsequently audited, the agency reimburses the overpaid tax (this assumption is not crucial, see Section 5b). It is assumed that both types of the taxpayer suffer losses, $\phi>0$ if audited ${ }^{7}$. For the agency carrying out audits is also costly, and for simplicity we assume that these costs, $c>0$, are constant. Revenues of the tax agency always consist of taxes paid plus the fine, in case it applies, minus the costs of audits, if they are carried out.

A strategy (pure or mixed) of player $i \in\{1,2\}$ will be denoted by $s_{i}$. It is not hard to see that both players will have four pure strategies. A pure strategy of the taxpayer has the form $\tau^{\prime} \tau^{\prime \prime}$, where $\tau^{\prime}, \tau^{\prime \prime} \in\{Y, y\}$. A pure strategy of the agency has the form $v w$, where $v, w \in\{a, d\}$. Taxpayer's strategy $\tau^{\prime} \tau^{\prime \prime}$ should be read as 'report $\tau^{\prime}$ when Nature selects type is $Y$ and report $\tau^{\prime \prime}$ if Nature draws type $y$ '. Similarly, the strategy $v w$ is interpreted as 'carry out $v$ if report is $Y$, and perform $w$ if report is $y$ '. Normally, a randomized strategy of a player is written as a probability distribution over the set of his pure strategies. We, however, will rather

\footnotetext{
${ }^{6}$ All our results do not depend on whether it is feasible to pay a high tax from low income at all, i.e. whether $y \geq Y t$ holds or not.

${ }^{7}$ This assumption leads to some surprising conclusion. We relax it later on, see subsection 3.3.
} 
focus on how each type of the taxpayer behaves and with what probability the agency audits each possible report, i.e. on players' behavioral strategies. This makes the exposition more transparent and intuitive ${ }^{8}$. In case the behavioral strategy $s_{i}$ is mixed, probabilities with which two different actions are selected will be given in round brackets.

The above setup represents a typical Bayesian game with observable actions. The solution concept usually employed for games of this type is that of perfect Bayesian Nash equilibrium. In order to use it, one needs to specify, so called, off-equilibrium beliefs of the agency. Those will be written as $\mu\left(\tau^{\prime} \mid \tau^{\prime \prime}\right)$, which should be read as 'probability that the true type is $\tau^{\prime}$ when the report is $\tau^{\prime \prime}$.

Players' payoffs, conditional on the report of the taxpayer, are summarized in the following two tables.

\begin{tabular}{|c|c|c|}
\hline & audit & don't \\
\hline$\tau=Y$ & $Y(1-t)-\phi, Y t-c$ & $Y(1-t), Y t$ \\
\hline$\tau=y$ & $y(1-t)-\phi, y t-c$ & $y-Y t, Y t$ \\
\hline
\end{tabular}

Figure 1: Taxpayer's report is $Y$.

\begin{tabular}{c|c|c|}
\multicolumn{1}{c}{ audit } & don't \\
\cline { 2 - 3 }$\tau=Y$ & $Y(1-t)-\theta(Y-y)-\phi, Y t+\theta(Y-y)-c$ & $Y-y t, y t$ \\
\cline { 2 - 3 }$\tau=y$ & $y(1-t)-\phi, y t-c$ & $y(1-t), y t$ \\
\hline
\end{tabular}

Figure 2: Taxpayer's report is $y$.

(Observe that each row in the above payoff matrices is the choice of income type by Nature and is not the strategy of the taxpayer. The latter is the choice of a particular matrix.)

Without loss of generality we also assume that while indifferent, the agency always prefers to audit and the taxpayer to cheat. Furthermore,

\footnotetext{
${ }^{8}$ All results, of course, can be stated in the conventional manner as well.
} 
we allow that $(Y-y)(t+\theta)>c$ holds. Otherwise iterative elimination of dominated strategies leads to a unique outcome, in which both types of the taxpayer report $y$, and the agency never carries out audits, no matter what the reports are.

It is essential to note that von Neumann-Morgenstern utilities of the players are identified with their monetary payoffs, thus all parties are assumed to be risk neutral. For the agency this assumption is typical, whereas it appears that the taxpayer is rather risk averse. However, Section $5 \mathrm{~h}$ will argue that there is no loss of generality in supposing that the taxpayer is also risk neutral in the present context.

A quick analysis of Figures 1 and 2 leads to the conclusion that after report $Y$ the dominating strategy of the agency becomes $d$, that is pure strategies $a a$ and $a d$ are strictly dominated. Moreover, report $y$ is audited if and only if the prior $\pi$ on $Y$ is sufficiently high, i.e.

$$
\pi \geq \frac{c}{(Y-y)(t+\theta)} \equiv \widetilde{\pi}
$$

Due to the assumption made, $1>\tilde{\pi}$. To make matters interesting, in what follows we will always assume that $1>\pi \geq \tilde{\pi}$. The first part of this inequality is strict, for otherwise the game becomes trivial, the same concerns the case $\pi<\widetilde{\pi}$, see Section 5i. Throughout, we will refer to the above setup as the basic model.

The game has the following two perfect Bayesian equilibria.

(i) Pooling on $Y$ :

$$
\begin{array}{ll}
s_{1}(\tau)=Y, & \text { for all } \tau \in\{Y, y\}, \\
s_{2}(Y)=d, & s_{2}(y)=(\beta, 1-\beta) .
\end{array}
$$

Where the probability with which the agency audits report $y$ enjoys

$$
1 \geq \beta \geq \max \left\{\frac{(Y-y) t}{(Y-y)(t+\theta)+\phi}, \frac{(Y-y) t}{\phi}\right\}=\frac{(Y-y) t}{\phi} .
$$

This equilibrium is supported by the following off-equilibrium beliefs of the agency: $\mu(Y \mid y) \in[\widetilde{\pi}, 1]$. In words, both types of the taxpayer report $Y$, and such reports are not audited. In case a low report $y$ is submitted, the agency audits it with probability at least $\frac{(Y-y) t}{\phi}$, since it thinks that it has come from type $Y$ with probability of at least $\tilde{\pi}$. The necessary condition for pooling is that $\beta \in[0,1]$, which implies $\phi \geq(Y-y) t$, i.e. that personal costs of audit are high enough. Also note how the assumption about players' strategies in case they are indifferent enters here: when $\phi=(Y-y) t$ type $y$ of the taxpayer is indifferent between 
reporting honestly and over-reporting his income, however, by assumption, the taxpayer cheats when indifferent, and this preserves the pooling equilibrium.

(ii) Semipooling:

$$
\begin{array}{ll}
s_{1}(y)=y, & s_{1}(Y)=\left(\alpha^{\prime}, 1-\alpha^{\prime}\right), \\
s_{2}(y)=\left(\beta^{\prime}, 1-\beta^{\prime}\right), & s_{2}(Y)=d .
\end{array}
$$

That is, type $y$ always reports honestly and type $Y$ cheats with probability $\alpha^{\prime}$. The agency audits low report $y$ with probability $\beta^{\prime}$ and does not audit report $Y$ at all.

The above $\alpha^{\prime}$ and $\beta^{\prime}$ are given by

$$
\alpha^{\prime}=\frac{1-\pi}{\pi} \frac{c}{(Y-y)(t+\theta)-c},
$$

or, using the definition of $\tilde{\pi}$, simply

$$
\alpha^{\prime}=\frac{1-\pi}{\pi} \frac{\tilde{\pi}}{1-\widetilde{\pi}}
$$

As for $\beta^{\prime}$, we have

$$
\beta^{\prime}=\frac{(Y-y) t}{(Y-y)(t+\theta)+\phi} .
$$

In the calculation of $\alpha^{\prime}$ and $\beta^{\prime}$ we used the fact that if a (type of) player randomizes, then he has to be indifferent, given the strategy of the opponent. It is straightforward to show that $\alpha^{\prime} \leq 1$ and $\beta^{\prime} \leq 1$. Observe that there is no equilibrium, in which the strategy of the agency upon receiving the signal $y$ would be

$$
\beta \in\left[0, \frac{(Y-y) t}{(Y-y)(t+\theta)+\phi}\right) \bigcup\left(\frac{(Y-y) t}{(Y-y)(t+\theta)+\phi}, \frac{(Y-y) t}{\phi}\right) .
$$

Note the following interesting things about the semipooling equilibrium. First, some taxpayers (type $y$ ) always report honestly, and some (type $Y$ ) cheat with positive probability. The agency, however, being unable to distinguish between the two types, audits everybody, who reports $y$ with the same probability $\beta^{\prime}$. Moreover, we will see that this is the only equilibrium, in which the agency carries out audits!

Second, although type $Y$ in fact 'forces' this equilibrium, he does not derive any gain in the utility sense from doing so, since the equilibrium strategy of the agency makes him indifferent between deviating and reporting honestly. Thus, in equilibrium the taxpayer cannot benefit from cheating! However, due to audits, both types of the taxpayer suffer losses, furthermore, the agency also bears the costs of audit. Even intuitively, 
this equilibrium is not socially optimal, i.e. Pareto dominated, and below we will show formally when this is the case.

It is instructive to find players' expected payoffs in these equilibria. In the pooling one,

$$
\begin{aligned}
& u_{1}^{P}=\underbrace{[\pi Y+(1-\pi) y](1-t)}_{\text {after-tax income }}-\underbrace{(1-\pi)(Y-y) t}_{\text {loss due to over-reporting }} . \\
& u_{2}^{P}=Y t .
\end{aligned}
$$

In the semipooling equilibrium we have

$$
\begin{aligned}
& u_{1}^{S P}=\underbrace{[\pi Y+(1-\pi) y](1-t)}_{\text {after-tax income }}-\underbrace{(1-\pi) \phi \beta^{\prime}}_{\text {loss due to audits }} . \\
& u_{2}^{S P}=y t+\pi t\left(1-\alpha^{\prime}\right)(Y-y) .
\end{aligned}
$$

It is intuitive that taxpayer's losses due to audits are smaller than those due to over-reporting, since

$$
(Y-y) t>\phi \beta^{\prime} \text {. }
$$

holds no matter whether $(Y-y) t \geq \phi$ or not. Therefore,

$$
u_{1}^{S P}>u_{1}^{P} .
$$

For the agency, however, the opposite is true since

$$
Y t>y t+\pi t\left(1-\alpha^{\prime}\right)(Y-y)
$$

reduces to

$$
1>\pi\left(1-\alpha^{\prime}\right) .
$$

Thus

$$
u_{2}^{P}>u_{2}^{S P} .
$$

Hence, there is a conflict of interests in the game in the following sense: the agency prefers the pooling equilibrium and the taxpayer - the semipooling one. Such situations are typical in game theory; however, an unusual feature of this model is that one party, namely the agency, can induce its favorite outcome by simply announcing with what probability it is going to audit report $y$, provided $\phi \geq(Y-y) t$. The costs of this threat to the agency do not matter, since in equilibrium no deviation occurs. 
Notice that if the agency aimed at maximizing its revenue, then its optimal strategy $\beta^{*}$ of auditing report $y$ would depend on $\phi$ in the following way

$$
\beta^{*}(\phi)= \begin{cases}\frac{(Y-y) t}{\phi}, & \text { if } \phi \geq(Y-y) t \\ \beta^{\prime}, & \text { otherwise. }\end{cases}
$$

Of course, any $\beta \in\left[\frac{(Y-y) t}{\phi}, 1\right]$ would be optimal in the case of pooling on $Y$; moreover, the precise value of such $\beta$ does not matter, since it is an off-equilibrium path. Nevertheless, we prefer to write $\beta^{*}(\phi)=\frac{(Y-y) t}{\phi}$ as equality, as if the agency were to minimize potential costs of audits. Similar reasoning will apply to formula (3.8) below.

\section{Introducing Honesty}

There are many ways to account for the existence of honest taxpayers. Following suggestions from extensive psychological and empirical literature $^{9}$ on the subject, we allow that honesty is a social norm. Dishonest behavior, thus, constitutes a deviation from the norm and inflicts on the deviators personal costs in the form of anxiety or regret. This can be modeled by adding a constant $\xi>0$ to taxpayer's payoffs in case he truthfully reports his income to the agency, i.e. when his strategy is $s_{1}(\tau)=\tau$ for all $\tau \in\{Y, y\}$. Such $\xi$ may be interpreted as a moral reward for being honest. Alternatively, we could assume that the taxpayer obtains $\xi$ uniformly, but the sense of 'guilt' reduces his utility by $\xi$ whenever cheating takes place. Of course, one may wonder, why to introduce honesty in exactly this way. Section $5 f$ discusses the rationale for doing this.

In general, one has a great freedom in specifying the precise conditions under which the taxpayer receives such a moral reward. Below we present just two intuitive cases that, of course, do not exhaust the range of opportunities. For convenience they are dubbed unconditional and selective honesty. Throughout the analysis, $\xi$ is assumed to be exogenously given and commonly known to the players, see Section $5 c$ for extensions.

\footnotetext{
${ }^{9}$ See, for instance, Torgler [19], [20] and references therein. Similar suggestions have been made in economic literature as well, eg. Spicer and Lundstedt [16].
} 
3.1. Unconditional Honesty. We will say that the taxpayer is unconditionally honest if he always receives moral reward in the amount of $\xi$, whenever he reports his type honestly, regardless the strategy of the agency. The resulting payoff matrices are displayed on Figures 3 and 4 .

\begin{tabular}{|c|c|c|}
\hline & audit & don't \\
\hline$\tau=Y$ & $Y(1-t)-\phi+\xi, Y t-c$ & $Y(1-t)+\xi, Y t$ \\
\hline$\tau=y$ & $y(1-t)-\phi, y t-c$ & $y-Y t, Y t$ \\
\hline
\end{tabular}

Figure 3: Taxpayer's report is $Y$.

\begin{tabular}{|c|c|c|}
\hline & audit & don't \\
\hline$\tau=Y$ & $Y(1-t)-\theta(Y-y)-\phi, Y t+\theta(Y-y)-c$ & $Y-y t, y t$ \\
\hline$\tau=y$ & $y(1-t)-\phi+\xi, y t-c$ & $y(1-t)+\xi, y t$ \\
\hline
\end{tabular}

Figure 4: Taxpayer's report is $y$.

There are three perfect Bayesian Nash equilibria now, of which the first two resemble the basic case of Section 2.

(i) Pooling on $Y$ :

$$
\begin{array}{ll}
s_{1}(\tau)=Y, & \text { for all } \tau \in\{Y, y\}, \\
s_{2}(Y)=d, & s_{2}(y)=(\beta, 1-\beta) .
\end{array}
$$

Where the probability with which the agency audits report $y$ satisfies

$$
1 \geq \beta \geq \max \left\{\frac{(Y-y) t-\xi}{(Y-y)(t+\theta)+\phi}, \frac{(Y-y) t+\xi}{\phi}\right\}=\frac{(Y-y) t+\xi}{\phi} .
$$

Again, the equilibrium is supported by the off-equilibrium beliefs $\mu(Y \mid y) \in$ $[\widetilde{\pi}, 1]$. The necessary condition for pooling is that personal costs of audit are high enough, i.e.

$$
\phi \geq(Y-y) t+\xi \text {. }
$$


(ii) Semipooling:

$$
\begin{array}{ll}
s_{1}(y)=y, & s_{1}(Y)=\left(\alpha^{\prime}, 1-\alpha^{\prime}\right), \\
s_{2}(y)=\left(\beta^{\prime \prime}, 1-\beta^{\prime \prime}\right), & s_{2}(Y)=d .
\end{array}
$$

Thus, type $y$ reports honestly, and type $Y$ cheats with probability $\alpha^{\prime}$, as defined in (2.2). The agency carries out audits of every report $y$ with probability

$$
\beta^{\prime \prime}=\frac{(Y-y) t-\xi}{(Y-y)(t+\theta)+\phi},
$$

and does not audit report $Y$. It is clear that to support this equilibrium we need to have

$$
(Y-y) t \geq \xi
$$

Note that in the previous case the optimal strategy of the agency in the semipooling equilibrium was to audit with a higher probability, i.e. $\beta^{\prime}>\beta^{\prime \prime}$. This is an intuitive conclusion. Thus the social loss in this equilibrium due to audits will be lower now.

(iii) Separating:

$$
\begin{aligned}
& s_{1}(\tau)=\tau, \\
& s_{2}(\tau)=d,
\end{aligned}
$$

for all $\tau \in\{Y, y\}$. The necessary condition for this equilibrium to hold is given by

$$
\xi>(Y-y) t
$$

(Recall that in case of equality, type $Y$ is indifferent and, thus, by assumption, cheats). This condition serves as an incentives compatibility constraint for both types of the taxpayer, precluding them from misreporting their income. In the sequel, we will refer to (3.5) as the honesty condition. Observe that it does not depend on the rate of fine ${ }^{10} \theta$, prior $\pi$, costs of audit $\phi$ and $c$.

Let us compare players' payoffs in these equilibria. In the pooling equilibrium

$$
\begin{aligned}
& u_{1}^{P}(\xi)=\underbrace{[\pi Y+(1-\pi) y](1-t)+\pi \xi}_{\text {after-tax income }}-\underbrace{(1-\pi)(Y-y) t}_{\text {loss due to over-reporting }} . \\
& u_{2}^{P}(\xi)=u_{2}(P)=Y t .
\end{aligned}
$$

Note that $\pi \xi$ reflects the fact that only type $Y$ obtains $\xi$, since type $y$ does not get $\xi$ in case of over-reporting.

\footnotetext{
${ }^{10}$ This is true even for a more general configuration of punishing schemes, see Section 5b.
} 
In the semipooling

$$
\begin{aligned}
& u_{1}^{S P}(\xi)=\underbrace{[\pi Y+(1-\pi) y](1-t)+\xi}_{\text {after-tax income }}-\underbrace{(1-\pi) \phi \beta^{\prime \prime}}_{\text {loss due to audits }} . \\
& u_{2}^{S P}(\xi)=y t+\pi t\left(1-\alpha^{\prime}\right)(Y-y) .
\end{aligned}
$$

Observe that in the semipooling equilibrium taxpayer's expected payoff is such as if both of its types received moral reward $\xi$, despite the fact that type $Y$ still cheats in equilibrium with probability $\alpha^{\prime}>0$. Moreover, since the agency's payoffs are not changed, the optimal mixed strategy of type $Y$ is the same as in the pooling equilibrium without $\xi$, and it yields the same expected payoff to the agency. Thus,

$$
u_{2}^{S P}(\xi)=u_{2}^{S P} .
$$

Finally, in the separating equilibrium we have

$$
\begin{aligned}
& u_{1}^{S}(\xi)=[\pi Y+(1-\pi) y](1-t)+\xi . \\
& u_{2}^{S}(\xi)=[\pi Y+(1-\pi) y] t .
\end{aligned}
$$

and no cheating or audits take place.

It is easily shown that

$$
\begin{aligned}
& u_{1}^{S}(\xi)>u_{1}^{S P}(\xi)>u_{1}^{P}(\xi) . \\
& u_{2}^{P}(\xi)>u_{2}^{S}(\xi)>u_{2}^{S P}(\xi) .
\end{aligned}
$$

Paralleling (2.6), the revenue-maximizing strategy of auditing report $y$ becomes

$$
\beta^{*}(\xi, \phi)= \begin{cases}\frac{(Y-y) t+\xi}{\phi}, & \text { if } \phi \geq(Y-y) t+\xi \\ \beta^{\prime \prime}, & \text { if } \phi<(Y-y) t+\xi \text { and } \xi \leq(Y-y) t \\ 0, & \text { if } \phi<(Y-y) t+\xi \text { and } \xi>(Y-y) t\end{cases}
$$

Graphically all equilibrium cases can be shown in the $\xi \times \phi$ plane as follows. 


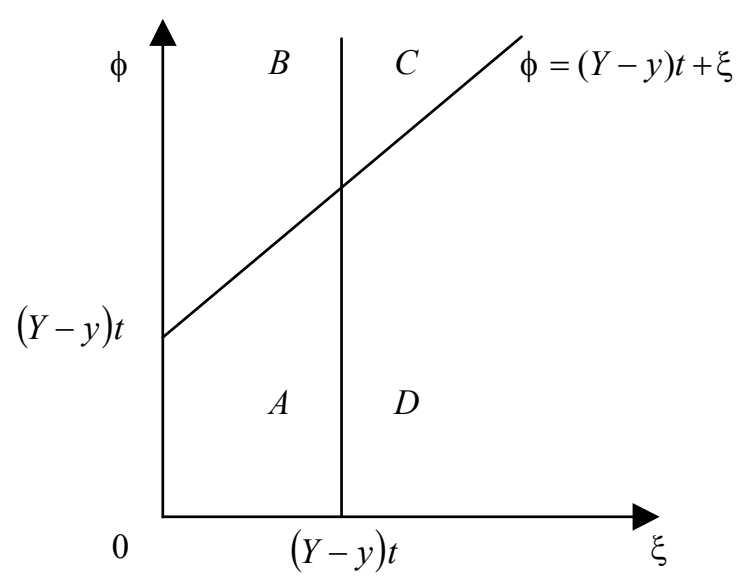

Figure 5: Admissible equilibria of the game.

Pooling equilibria occur in regions $B$ and $C$; semipooling in regions $A$ and $B$; finally separating equilibria in $C$ and $D$. Thus, separating and semipooling are mutually exclusive types of equilibria, and these two are the only mutually exclusive types out of the three. According to (3.8), the agency will set $\beta^{*}=\frac{(Y-y) t+\xi}{\phi}$ in $B$ and $C, \beta^{*}=\beta^{\prime \prime}$ in $A$, and $\beta^{*}=0$ in $D$.

An interesting variant of unconditional honesty arises when the taxpayer obtains $\xi$ even in the case of over-reporting. This modification, however, changes only the off-equilibrium strategy of the agency in the pooling equilibrium. Namely, the probability of auditing low report $y$ becomes independent of $\xi$ and is the same as in (2.1). The agency's beliefs supporting the equilibrium are $\mu(Y \mid y) \in[\widetilde{\pi}, 1]$, and revenue comparisons expressed by formulae (3.6) and (3.7) remain valid.

3.2. Selective Honesty. Let us suppose now that the taxpayer receives moral reward for paying honestly if and only if (i) his strategy is $s_{1}(\tau)=\tau$ for all $\tau \in\{Y, y\}$, and (ii) such report is not audited. Alternatively, we could suppose that once the agency erroneously audits an honest taxpayer, the latter suffers 'moral' costs of $-\xi$ in addition to the auditing costs $\phi$. Formally, the payoff matrices now become as in Figures 3 and 4 with the difference that $\xi$ is removed from the taxpayer's payoffs, once the agency selects audit. All other respects of the model are not changed.

As in the previous subsection, the game has three types of equilibria. Semipooling and separating equilibria are exactly the same as before. However, necessary conditions for the pooling equilibrium slightly change. Now the agency should threaten to audit all reports $y$ with 
probability $\widehat{\beta}$, given by

$$
1 \geq \widehat{\beta} \geq \max \left\{\frac{(Y-y) t-\xi}{(Y-y)(t+\theta)+\phi}, \frac{(Y-y) t+\xi}{\phi+\xi}\right\}=\frac{(Y-y) t+\xi}{\phi+\xi} .
$$

A necessary condition for this pooling equilibrium is again $\phi \geq(Y-y) t$. Once it holds, it is easy to show that

$$
\frac{d}{d \xi} \widehat{\beta} \geq 0 \text {. }
$$

This conclusion is not surprising, since when taxpayers have higher moral reward for reporting honestly, more severe threats of the agency are needed in order to discourage the taxpayer of type $y$ from truthful revelation. Note, that the same off-equilibrium beliefs of the agency $\mu(Y \mid y) \in$ $[\widetilde{\pi}, 1]$ support this equilibrium.

As far as expected payoffs are concerned, in the pooling and separating equilibrium nothing changes as compared to the case of unconditional honesty. In the semipooling equilibrium, however, the agency receives the same payoff, whereas the taxpayer now suffers not only auditing $\operatorname{costs} \beta^{\prime \prime} \phi(1-\pi)$, but also 'moral' ones in the amount $\beta^{\prime \prime} \xi(1-\pi)$. As a consequence, for the taxpayer the payoff difference between pooling and semipooling equilibria disappears!

3.3. Aggregate Effects of Honesty. Let us summarize how the introduction of honesty affected the basic model. First, it follows that there are no absolutely honest taxpayers. Suppose that the honesty condition defined in (3.5) holds. Then for each honesty parameter $\xi$ we may find a tax level $\bar{t}$ such that any tax rate $t>\bar{t}$ will violate (3.5) and lead to evasion (for instance, semipooling equilibrium). Hence, up to a certain level people enjoy being honest, above this level they start behaving opportunistically. Correspondingly, the model loses its separating equilibria. Although this conclusion is straightforward, other models dealing with honest taxpayers, eg. Erard and Feinstein [5], do not reach it.

Although the agency's payoffs do not explicitly depend on the existence of moral reward, the agency can be made better off provided $\phi<(Y-y) t$ and $\xi>0$ hold. This can be seen as follows. Once $\phi$ is sufficiently small, the pooling equilibrium is not possible any longer. In the basic setup the only remaining equilibrium is semipooling. However, when honesty is introduced in the analysis and (3.5) holds, the separating equilibrium becomes possible. In this equilibrium the agency's expected payoff is higher than in the case of semipooling. Moreover, the taxpayer's 
utility is always higher in the separating equilibrium as compared to the semipooling one. This exemplifies how audits may not be optimal from the welfare point of view. In such cases enhancing honesty seems to be a desirable policy.

The same conclusion, of course, would be reached if from the very beginning we assumed that $\phi=0$. Recall that $\phi>0$ led to a somewhat surprising result, namely it was possible that type $y$ reported more than it was due. To some the possibility that an honest taxpayer reports more than it is needed may not seem reasonable. Hence, by ruling it out and imposing $\phi=0$ one basically obtains the same result about positive welfare effects of honesty.

3.4. Comparative Statics. We conclude this Section with a brief discussion of the comparative statics effects on the equilibrium strategies along the equilibrium path derived in subsections 3.1 and 3.2. By assuming that inequalities (3.2) and (3.4) hold strictly, we can investigate how small changes in the exogenous parameters of the model $\pi, \theta, \xi, \phi, t, c$ and the income levels $Y, y$ affect the equilibrium strategies of the players. Since in all formulae it is not the absolute level of income, but the difference $Y-y$ is the one that is important, in what follows we define $x:=Y-y$.

In both pooling and separating equilibria players' strategies are pure, thus infinitesimal changes of the above exogenous variables do not have any effect on the equilibrium strategies. For the semipooling equilibrium the situation is different, because it involves mixing. Using formulae (2.2) and (3.3) we can write optimal strategies $\alpha^{\prime}$ and $\beta^{\prime \prime}$ as functions $\alpha^{\prime}(\pi, c, \theta, t, x)$ and $\beta^{\prime \prime}(\xi, \phi, \theta, t, x)$. Then the following relations can be easily checked.

$$
\begin{aligned}
& \frac{d}{d \pi} \alpha^{\prime}<0, \quad \frac{d}{d c} \alpha^{\prime}>0, \quad \frac{d}{d \theta} \alpha^{\prime}<0, \quad \frac{d}{d t} \alpha^{\prime}<0, \quad \frac{d}{d x} \alpha^{\prime}<0 . \\
& \frac{d}{d \xi} \beta^{\prime \prime}<0, \quad \frac{d}{d \phi} \beta^{\prime \prime}<0, \quad \frac{d}{d \theta} \beta^{\prime \prime}<0, \quad \frac{d}{d t} \beta^{\prime \prime}>0, \quad \frac{d}{d x} \beta^{\prime \prime}>0 .
\end{aligned}
$$

Whereas $c, \pi, \theta, \xi$ and $\phi$ have somewhat expected effects on $\alpha^{\prime}$ and $\beta^{\prime \prime}$, the same cannot be said about $t$ and $x$. Intuitively, the higher the income dispersion or the tax rate, the more 'profitable' is evasion and hence $\alpha^{\prime}$ should grow. However, the above formulae predict precisely the opposite. The reason for this is that the semipooling equilibrium involves mixed strategies. Thus, equilibrium probabilities with which 
players select their pure strategies depend not on their own payoffs, but on the payoffs of the opponent. Hence, the more 'beneficial' is evasion, the higher is the probability of audits, and, as a response to this, the lower is the probability of evasion.

\section{Equilibrium Refinements}

To narrow down the set of admissible equilibria one can employ a refinement. Specifically, we will use the following three: the intuitive criterion by Cho and Kreps [4], evolutionary stability in the spirit of Swinkels [18], and stochastic stability in the dynamic environment called adaptive play proposed in Young [23]. To safe space we will just focus on the results. Details are left out and the interested reader should consult the original papers if necessary.

The intuitive criterion rules out 'unreasonable' beliefs of the agency at those information sets that are not reached in equilibrium. In the basic model developed in Section 2 both pooling and semipooling equilibria satisfy the intuitive criterion, as is easy to check. This is still so for the models in subsections 3.1 and 3.2, provided the separating equilibrium is not possible, i.e. if (3.5) does not hold. What we will show now is that if the separating equilibrium is possible (i.e. the honesty condition holds), then it is the only equilibrium, which passes the intuitive criterion test.

First, as we noted earlier, separating and semipooling equilibria are mutually exclusive. Thus, in the presence of the separating equilibrium, semipooling does not obtain. Second, suppose that initially pooling on $Y$ is the case. This equilibrium is supported by beliefs $\mu(Y \mid y) \in[\widetilde{\pi}, 1]$. However, if $\xi>(Y-y) t$, then type $Y$ never reports $y$. Thus the agency upon seeing the off-equilibrium signal $y$ should reason that it could have been sent only by type $y$, and must not audit such a report. In other words, given $\xi>(Y-y) t$, the only reasonable off-equilibrium beliefs are $\mu(Y \mid y)=0$. As for type $y$, he indeed would like to report $y$, since it earns him as much as $y(1-t)+\xi$, which is strictly larger than the equilibrium payoff $y-Y t$. Hence, given the presence of the honesty condition, pooling on $Y$ does not survive. We can show these effects graphically: on Figure 5 it would imply that the admissible region of the pooling equilibrium does not extend beyond the vertical line $\xi=(Y-y) t$, regardless of the value of $\phi$. That is, pooling is confined to region $B$ only. 
In order to investigate the evolutionary stability of the model, one can apply a multipopulation ${ }^{11}$ evolutionary approach (see Weibull [21], Chapter 5). The logic behind this type of refinement is that the game is assumed to be played not by (in our case) two players, but two infinite ${ }^{12}$ populations of taxpayers and agencies. Furthermore, the population of taxpayers is subdivided in two classes, corresponding to the two types of income, whose relative proportions are $\pi$ and $1-\pi$. Every member of every population is programmed to play a specific pure strategy. At each point in time one taxpayer and one agency are randomly drawn from their populations and play the game. It is well know that mixed Nash equilibria are not evolutionary stable, since players have multiple best replies and each population can be invaded by mutants who earn the same expected payoffs as the incumbents. Hence, the semipooling equilibrium automatically fails the test for evolutionary stability.

The pooling equilibrium is pure, however, it is not evolutionary stable either. The reason is that the signal $y$ is not sent in this equilibrium, therefore any response of the agency to $y$ is optimal. In particular, members of the population of agencies can costlessly decrease their probability $\beta$ of auditing reports $y$. However, once $\beta \leq \frac{(Y-y) t+\xi}{\phi}$, the taxpayer of type $y$ will deviate from the equilibrium by sending his truth-telling signal $y$. Finally, it is an easy exercise to check that player' strategies in the separating equilibrium are evolutionary stable, since such an equilibrium is strict. By Proposition 5.1 in Weibull [21] we obtain that this is the only evolutionary stable equilibrium of the game.

The notion of stochastic stability is designed to capture stability in dynamic situations when players can observe and analyze a sample of past plays in order to predict future behavior of their opponents. All players are assumed to be rational, in the sense that they compute their best responses correctly with probability close to one. However, with small probability they select their strategies at random, and hence may make mistakes. To check stochastic stability in our game we can apply the results of Jacobsen et al. [10]. By Theorem 1 there, the only stochastically stable equilibrium will be the separating one.

\footnotetext{
${ }^{11}$ Here one population represents taxpayers and the other population - the set of identical tax agencies (or the set of servants in the tax administration).

${ }^{12}$ Not 'big and finite', but infinite - one really needs a continuum of players. What happens if the size of populations is finite see Slafer [15] and Weibull [21] p. 43.
} 


\section{Discussion}

(a) Can taxpayers have more than two types of income? Certainly so. However, recall that our main purpose was to single out 'local' and individualistic, not global effects of the interaction between taxpayers and the agency. We can imagine that a particular taxpayer, say a business firm, files a declaration to a servant in the tax administration, who is more or less familiar with the type of income that such kind of firm generally earns. In this situation two income levels are enough to capture the tension between the parties. The model can be made more 'realistic' by including many types or even a continuum thereof. Nevertheless, this increased mathematical complexity does not seem to bring any significant payoff in terms of intuition.

(b) The fine may be levied in many ways. For instance, it may be proportional to the amount of evaded tax, not income, or be lump-sum. In the present setting with fixed levels of income and taxes all these modifications are equivalent. In a more general setup, for instance if different types of income are subject to different tax rates, specifications of the fine do matter. However, the difference will concern only randomized strategies in the semipooling equilibrium and off-equilibrium beliefs and strategies of the agency in the pooling one. As for our results about the structure of equilibria and payoff comparisons, they remain unaltered. Similarly, the agency may not reimburse the overpaid tax to type $y$ in case it audits him, since this is anyway an off-equilibrium path.

(c) In the present analysis we took the amount of moral reward for an honest type of the taxpayer as an exogenous variable. It can be made endogenous in many ways. For instance, one could suppose that $\xi$ negatively depends on the probability of audits. This may be interpreted as if taxpayers who are audited more often behave more opportunistically towards the agency. We do not make $\xi$ endogenous for the same reason that we did not include more than two types of the taxpayer in the present model. Such an extension is expected to yield simply a different value for the optimal probability of auditing report $y$. It will not change the nature of equilibria or their welfare properties in any relevant way. In fact, the reader can easily verify that the three equilibria we have derived are the only possible types of equilibria in this tax evasion game, regardless of the way honesty is introduced. Combining this with Section 5b allows us to conclude that the overall logic of the model is stable under some modifications.

(d) Without honesty, separating equilibrium is not possible in the basic setup. One way of making it possible would be to adjust the model so 
that different reports are subject to different tax rates, then for some values of parameters we will have separation. Specifically, if $Y$ and $y$ are taxed by the rates $T$ and $t$, respectively, then the separating equilibrium arises whenever $Y T<y t$ holds. This implies $T<t$, i.e. the tax system must be regressive. However, condition $T<t$ is not reasonable if both types relate to the same income category or if we deal with corporate taxation, which in many countries uses either uniform or progressive tax rate.

Another way to enforce separation would be to adopt the following auditing strategy of the agency. Suppose that if an audited taxpayer turns out to be honest (i.e. his report $\tau$ turns out to be equal to his true type, which is found out during the audit, by assumption), the agency gives him a (monetary) bonus that has some positive utility. It can be shown that for some range of parameters, though not uniformly, such strategy would make it incentive compatible to report honestly. The problem is that this strategy is not incentive compatible for the agency, at least in the current setup, since the latter will never audit report $Y$.

(e) We aggregated expected utilities of both types of the taxpayer with the same weight. Whenever this does not seem a plausible assumption, our conclusions about payoff comparison may be invalid. Nevertheless, it appears to be generally accepted that all types of a player receive the same importance.

(f) Some may argue that, perhaps, it is not only inner honesty and some positive $\xi$ but something else that is also responsible for high compliance. For instance, factors like social stigma - based on the presumption that evading taxpayers, if detected, will be ostracized - can also explain truthful revelation. This, however, is only partially true. Specifically, costs inflicted on evaders by the society, in case dishonest behavior is detected, cannot fully account for taxpayer's compliance. It can be shown $^{13}$ that in order to make truthful reporting self-enforcing for type $Y$, one somehow needs to increase his payoff after report $Y$ is sent, decreasing payoff after report $y$ does not achieve the same result! In other words, increasing the size of punishment leads only to a lower probability of evasion for type $Y$ in the semipooling equilibrium, but it can never eliminate evasion completely, as long as the taxpayer is an expected utility maximizer.

\footnotetext{
${ }^{13}$ Formally, construct a $2 \times 2$ payoff matrix, in which taxpayer's pure strategies are $y y$ and $Y y$, and that of the agency are $d a$ and $d d$. Then observe that the only way to make the truth revealing strategy $Y y$ dominate the evasive strategy $y y$ is to increase taxpayer's payoff at the profile $(Y y, d d)$. This is exactly what the introduction of $\xi>0$ did.
} 
(g) We considered just two types of honest behavior and remarked that this did not cover all possible cases. Certainly, the agency does not need to know in which exactly way honesty enters taxpayer's considerations. We exclude this possibility and consider only situations, in which both parties have 'common knowledge' as to what game is played. This is not a serious drawback. Games in which some parameters (payoffs) are not commonly known can be modeled as games of incomplete information. The famous purification result in Harsanyi [9], implies that in the limit, when the amount of incomplete information tends to zero, each Nash equilibrium of the original game can be approximated as the limit of some sequence of equilibria of the modified game. Similarly we assume that both parties agree on the numerical value of $\pi$. This common prior assumption is also called Harsanyi doctrine. Its pros and cons are extensively discussed in Aumann [2].

(h) Let $s$ be a profile of strategies, $x_{i}(s)$ a monetary payoff of player $i$ when $s$ is played, and $X_{i}$ a set of all possible monetary payoffs that player $i$ can obtain in the game. Consider an increasing and continuous utility function $u_{i}: X_{i} \rightarrow \mathbb{R}$. It is well-known that if $u_{i}(\cdot)$ is concave, then player $i$ is risk averse. By applying $u_{i}\left(x_{i}(s)\right)$ instead of $x_{i}(s)$, we simply rescale the original monetary payoffs of player $i$. That is, the absolute difference between two monetary payoffs $x_{i}(s)$ and $x_{i}\left(s^{\prime}\right)$ becomes smaller in 'utility' terms, i.e. $\left|x_{i}(s)-x_{i}\left(s^{\prime}\right)\right|>\left|u_{i}\left(x_{i}(s)\right)-u_{i}\left(x_{i}\left(s^{\prime}\right)\right)\right|$. It is not hard to see that if under these conditions some profile $s$ is a pure strategy Nash equilibrium, then it remains to be an equilibrium even after the rescaling. As for the mixed equilibria, they will change, because payoffs have been rescaled in a non-linear way.

Therefore, in our game accounting for risk aversion of the taxpayer does not affect either pooling or separating equilibria. Nor does it change the welfare properties of all equilibria, as defined in (2.4) - (2.5) and (3.6) - (3.7). What it does change, though, are the optimal probabilities of auditing, $\beta^{\prime}$ and $\beta^{\prime \prime}$. However, their precise numerical values are not essential in the present context.

(i) We could also consider the case $\pi<\tilde{\pi}$, which is identical to supposing $(Y-y)(t+\theta) \leq c$. Instead of pooling on $Y$ and the semipooling equilibrium, we would then have just one equilibrium, in which all types of the taxpayer pool on report $y$, and the agency does not audit anybody. Once honesty is introduced and separation becomes possible, this equilibrium would be ruled out by the intuitive criterion, in the same way, as pooling on $Y$ was. In reality such a case cannot be dismissed as meaningless; indeed for some groups of taxpayers it would be relevant. We do 
not treat it in detail here because of its relative simplicity and limited applicability, as compared to the case $\pi \geq \tilde{\pi}$.

(j) The analysis presented in this paper should not provoke a misleading conclusion that game-theoretic models of tax evasion are superior to that of the first generation. The main difference between the two approaches is that in one case players take into account the consequences of their own actions on the actions of the opponents, and in the other case, they disregard them. We are strongly convinced that it is, first of all, a matter of empirical evidence to see if in such a complex dilemma filled with uncertainty, as evading/auditing decision in fact is, either taxpayers or the agency are willing to take each other's actions as random variables, to which subjective probability distributions are assigned. To the best of our knowledge, there have been no such empirical studies. Thus, either point of view can be questioned. However, as far as 'explanatory' power of the model is concerned, the fact that it 'fits' empirical data on high compliance in the presence of low rates of audits, it should be said that a first generation model would do equally well, if honesty was introduced there in the same way we did it here. Namely, high $\xi$ can be identified with significant levels of tax morale, so that it makes it incentive compatible for taxpayers to report their income honestly. Thereby, the optimal amount of evasion will be reduced and, perhaps, a much smaller and more realistic coefficient of risk aversion will be needed in order to be in line with empirical evidence. Nevertheless, those who still cite and test first generation models today, completely disregarding game-theoretic premises, do make a serious mistake!

(k) What does the model do and what does it not do? Let us summarize what has actually been achieved. We will start from limitations. First of all, the model does not predict a level of evasion in the aggregate. It would be silly to claim that taxpayers either report high income or evade with probability $\alpha^{\prime} \pi$, depending on the equilibrium. Similarly, stating that the agency either audits with probability $\beta^{\prime}\left(\beta^{\prime \prime}\right)$ all reports $y$ or does not audit them at all would not be wise. Players' adherence to their mixed equilibrium strategies rests on a very special assumption. Namely, it must be common knowledge that any deviations from the equilibrium strategies will be correctly exploited by the opponents ${ }^{14}$. In most situations in reality this is hardly the case. Moreover, the players may not have incentives to use their randomized strategies with equilibrium probabilities, since, given the correct strategy of the opponent, they are indifferent in utility terms. This may lead, for instance, to the

\footnotetext{
${ }^{14}$ For more on this and related topics, see Pruzhansky [12].
} 
agency playing its maximin strategy, that guarantees the same level of tax proceedings in expected terms after the signal $y$, no matter which type is sending it.

Any other 'aggregate' conclusions should similarly be avoided. The model did not intend to describe how a taxpayer representing the whole population interacts with the agency. Our aim was to emphasize particular features of the tax collecting process within specific groups of taxpayers. Groups that are commonly known by both players to be relatively homogenous with respect to such parameters as their true and evaded income. It follows that across groups the equilibrium strategies of both players may differ. That is, different groups may, in fact, coordinate on different Nash equilibria.

Finally, the reader should be aware of the 'unnatural' interpretation of randomized strategies in the context of a one-shot game. Biological interpretations of mixing as shares of populations playing different pure strategies with these shares corresponding to the probabilities $\alpha^{\prime}$ and, say, $\beta^{\prime \prime}$ are possible, though. However, thinking about mixing as the frequencies with which the players choose their pure strategies over time does not exactly fit in the present setup. Otherwise we should have modeled the repetition explicitly, taking into account such things as taxpayer's reputation and the fact that players do not disregard intertemporal links between plays. It appears to us that the best way to interpret randomized strategies would be to view them as beliefs that one party holds about possible actions of the other one. In this way the semipooling equilibrium models a stable social situation, in which players' beliefs form a Nash equilibrium (for a precise definition of the equilibrium in beliefs see Osborne and Rubinstein [11], pp. 43-44).

As far as the merits of the model are concerned, the following three points are worth mentioning. First, the importance of honesty per se is immediate. There is hardly anyone, who would claim the opposite. The model highlights this importance in a specific way, as welfare differences between semipooling and separating equilibria. Second, selective and unconditional honesty, being distinct modes of psychological behavior, do not differ much in terms of equilibrium payoffs or strategies. This suggests that even more general configurations of honesty may, in fact, lead to the same pattern of equilibrium strategies. Third, it appears that situations á la semipooling equilibrium arise in reality most often. That is, players' strategies (beliefs) oscillate around the values, specified by formulae (2.2), (2.3) and (3.3). Our analysis of comparative statics in subsection 3.4 provides a useful insight on how these strategies (beliefs) change with respect to the exogenous parameters of the model. 


\section{REFERENCES}

[1] Allingham, M., A. Sandmo (1972) "Income Tax Evasion: A Theoretical Analysis", Journal of Public Economics, 1, 323-38.

[2] Aumann, R. J. (1987) "Correlated Equilibrium as an Expression of Bayesian Rationality", Econometrica, 55, 1-18.

[3] Barro, R., X. Sala-i-Martin Economic growth, McGraw-Hill Inc. 1995.

[4] Cho, I., D. Kreps (1987) "Signaling Games and Stable Equilibrium", Quarterly Journal of Economics, 102, 179-221.

[5] Erard, B., J. Feinstein (1994) "Honesty and Evasion in the Tax Compliance Game", Rand Journal of Economics, 25, 1-19.

[6] Graetz, M., J. Reinganum, L. Wilde (1986) "The Tax Compliance Game: Toward an Interactive Theory of Law Enforcement", Journal of Law and Economic Organization, 2, 1-32.

[7] Frey, B. S. "The Role of Deterrence and Tax Morale in Taxation in the European Union", Jelle Zijlstra Lecture 1, Netherlands Institute for Advanced Study in the Humanities and Social Sciences 2003.

[8] Friedman, M. Essays in Positive Economics, Univ. of Chicago Press 1953.

[9] Harsanyi, J. (1973) "Games with Randomly Distributed Payoffs: A New Rationale for Mixed Strategy Equilibrium Points", International Journal of Game Theory, 2, 1-23.

[10] Jacobsen, H., M. Jensen, B. Sloth (2001) "Evolutionary Learning in Signaling Games", Games and Economic Behavior, 34, 34-63.

[11] Osborne, M., A. Rubinstein A Course in Game Theory, MIT Press 1994.

[12] Pruzhansky, V. (2004) "A Discussion of Maximin", Tinbergen Institute Discussion Paper.

[13] Reinganum, J., L. Wilde (1985) "Income Tax Compliance in a Principal-Agent Framework", Journal of Public Economics, 26, 1-18.

[14] Reinganum, J., L. Wilde (1986) "Equilibrium Verification and Reporting Policies in a Model of Tax Compliance", International Economic Review, 739-60.

[15] Shaffer, M. E. (1988) "Evolutionary Stable Strategies for Finite Population and a Variable Contest Size", Journal of Theoretical Biology, 132, 469-78.

[16] Spiser, M., S. Lundstend (1976) "Understanding Tax Evasion", Public Finance, 31, 295-305.

[17] Srinivasan, T. N. (1973) "Tax Evasion: A Model", Journal of Public Economics, $2,339-46$.

[18] Swinkels, J. (1992) "Evolutionary Stability With Equilibrium Entrants", Journal of Economic Theory, 57, 333-42.

[19] Torgler, B. (2002) "Speaking to Theorists and Searching for Facts: Tax Morale and Tax Compliance in Experiments", Journal of Economic Surveys, 16, 657-83.

[20] Torgler, B. (2003) "To Evade Taxes or Not to Evade: That Is the Question", Journal of Socio-Economics, 32, 283-302.

[21] Weibull, J. W. Evolutionary Game Theory, MIT Press 1997.

[22] Yitzhaki, S. (1974) "Income Tax Evasion: A Theoretical Analysis", Journal of Public Economics, 3, 201-2.

[23] Young, P. (1993) "Evolution of Conventions", Econometrica, 61, 57-84. 